\title{
Tumor-host interactions: the role of inflammation
}

\author{
Marie-Aude Le Bitoux • Ivan Stamenkovic
}

Accepted: 7 October 2008 / Published online: 25 October 2008

(C) The Author(s) 2008. This article is published with open access at Springerlink.com

\begin{abstract}
It is well established that interactions between tumor cells and the host tissue stroma play a key role in determining whether and how any given solid malignancy will develop. In most cases, tumor cells hijack stromal cell functions for their own benefit and ultimately dictate the rules of engagement to the host tissue microenvironment. However, the contribution of the different stromal cell components to tumor growth remains to be clarified. Because most solid tumors are accompanied by a local inflammatory response, it has long been thought that inflammation and carcinogenesis are related. If formal proof that cancer can be initiated by inflammation in the absence of exogenous carcinogens is still lacking, there is abundant evidence that the inflammatory response can play a central role in modulating tumor growth and progression. This review will discuss some of the mechanisms whereby inflammation can both enhance and inhibit tumor growth.
\end{abstract}

Keywords Carcinogenesis - Inflammation - Cytokines · Mediators · Tumor-associated macrophages · Lymphocytes

\section{Introduction}

It is becoming increasingly apparent that the tumor microenvironment plays a determining role in cancer

M.-A. Le Bitoux $\cdot$ I. Stamenkovic $(\square)$

Division of Experimental Pathology,

Institute of Pathology, CHUV,

Lausanne, Switzerland

e-mail: ivan.Stamenkovic@ chuv.ch

M.-A. Le Bitoux · I. Stamenkovic

Faculty of Biology and Medicine,

University of Lausanne, Lausanne, Switzerland development. Host tissue cells, including fibroblasts and other components of the cellular stroma are not passive bystanders in tumor growth and invasion but are active participants in the promotion of neoplastic propagation throughout the organism (Tlsty and Coussens 2006). Indeed, tumor growth depends, in large part, on the functional relationship between tumor and stromal cells, including, among others, macrophages, mast cells, adipocytes, and fibroblasts. Whenever carcinoma cells breach the natural barrier constituted by the basement membrane, they for the first time enter into direct physical contact with the host tissue stroma. The extent of what follows depends in part on the biological properties of the tumor cells and in part on the composition of the invaded stroma. However, tumor cells invariably cause a stromal reaction whose nature and amplitude vary according to the tumor type and a variety of individual traits.

A typical stromal reaction to neoplastic cells begins with the recruitment and activation of mesenchymal stem cells, endothelial cell precursors, fibroblasts, dendritic cells and a variety of leukocyte subsets (Olumi et al. 1999). Along with the tumor cells themselves, these different cell types secrete a variety of growth factors, chemokines and enzymes, that contribute to the ensuing tissue remodelling, as defined by the degradation of existing and deposition of new extracellular matrix (ECM) proteins and proteoglycans, and angiogenesis (Fig. 1). Together, these events resemble those that follow tissue injury, and have led to the description of cancer by Harold Dvorak as a "wound that never heals" (Dvorak 1986).

Stromal reaction to tissue injury, whether induced by trauma or infection, represents a defense mechanism of the organism that strives to limit the extent of the damage, eliminate the offending agent, if one is present, and restore the integrity of the tissues. Stromal reaction to invading 
Fig. 1 Representation of the inflammatory response to tumor growth

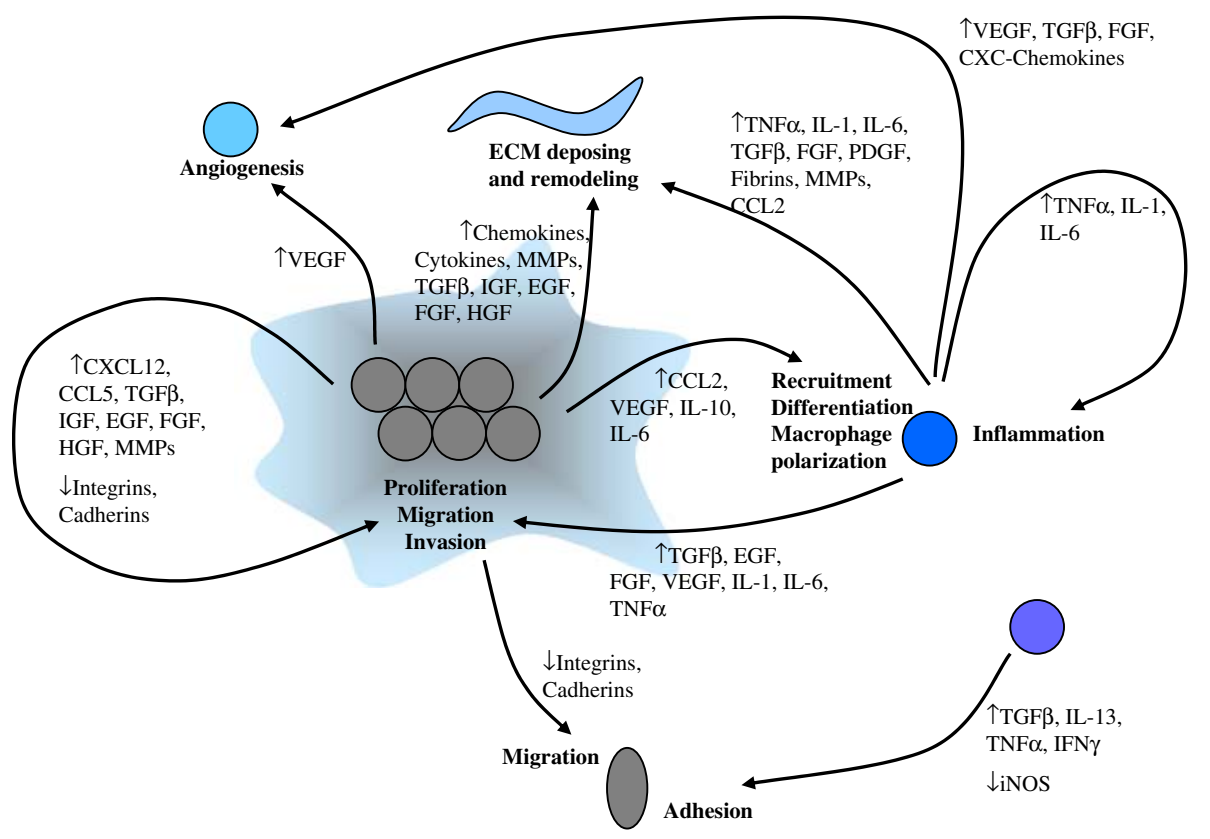

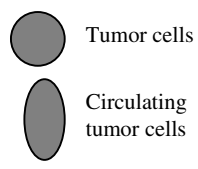

cancer cells may have at least two interpretations: similar to injury, it may constitute a defense mechanism to a pathological situation represented by the growth of aberrant cells that may mimic aspects of tissue injury; a more perverse interpretation would be that tumor cells harbour a program to elicit a stromal response that will facilitate tumor progression and dissemination as part of the tumor cell quest for survival. The correct interpretation probably lies somewhere in between the two-it is likely that the initial stromal response is indeed a defense mechanism that becomes subverted by the tumor cells, which can effectively counteract the growth inhibitory effects of the stromal reaction and harness its growth promoting effects for their own benefit.

Incubation of normal human fibroblasts with culture media conditioned by metastatic prostate cancer cell lines illustrates the importance of the effect of tumor cell-derived factors on the host tissue stroma (Kaminski et al. 2006). Tumor cell-conditioned culture media-stimulated fibroblasts are reported to display a phenotype reminiscent of carcinoma-associated fibroblasts (CAFs), secreting keratinocyte growth factor (KGF), tumor necrosis factor- $\alpha$ $(\mathrm{TNF} \alpha)$, basic fibroblast growth factor (bFGF), platelet derived growth factor (PDGF), hepatocyte growth factor (HGF) and vascular endothelial growth factor (VEGF), that can in turn stimulate proliferation of both normal and neoplastic cells. Conversely, fibroblast conditioned culture medium can enhance proliferation and anchorage-independent
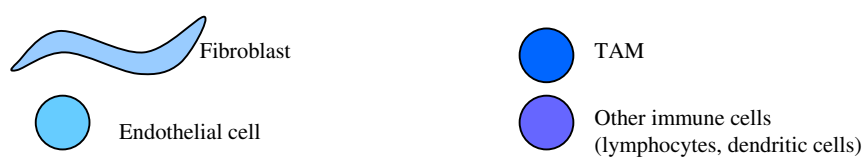

growth of some carcinoma cell lines and facilitates migration of others (Kaminski et al. 2006). CAFs have also been reported to stimulate transformation and progression of initiated prostate epithelial cells (Olumi et al. 1999).

Understanding how tumor cells control and benefit from host tissue responses could open the way toward designing therapeutic strategies targeting the molecular mechanisms that underlie relevant tumor-host interactions. Successful targeting of such mechanisms could result in severance of stromal support and functional isolation of tumor cells leading either to their demise or their sensitization to conventional and new therapies. A key issue, therefore, is to elucidate the mechanisms involved and to determine which events to target, bearing in mind that these may vary according to the tumor type and stage of progression. This review will focus on the role of the inflammatory response to tumor growth.

\section{A brief overview of inflammation}

Tissue injury, whether physical, chemical or infectious, triggers a sequence of events that constitute the inflammatory response. Inflammation is characterized by coordinated blood borne delivery to injured tissues of soluble mediators and cells that can eliminate the agent responsible for the injury and initiate tissue repair. Mast cells and tissue-resident 
macrophages are the first host tissue cells to become activated in response to injury and behave as orchestrators of the series of events that follow. Mast cells, which reside along vessel walls, are readily activated by crosslinking of cell surface $\mathrm{Fc}$ receptors and by $\mathrm{C} 3 \mathrm{a}$ and C5a fragments of the complement, generated by the presence of bacteria and/ or immune complexes. Activated mast cells rapidly release preformed vasoactive amines, principally histamine, which determine the early vascular response, characterized by a transient arteriolar constriction followed by capillary and venular dilatation and augmented endothelial permeability. Macrophages are also activated by Fc receptor crosslinking, $\mathrm{C} 3 \mathrm{a}$ and $\mathrm{C} 5 \mathrm{a}$, as well as mediators released by injured cells and the F-Met-Leu-Phe peptide of bacterial cell walls. Activated macrophages produce a host of cytokines, chief among which are interleukin-1 (IL-1), TNF $\alpha$ and IL-6. IL-1 and TNF $\alpha$ have similar and synergistic effects on vascular endothelial cells that include endothelial cell activation and expression of E- and P-selectin as well as intercellular adhesion molecule-1 (ICAM-1) on the cell surface. E- and $\mathrm{P}$-selectin recognize sialylated and fucosylated oligosaccharides on E-selectin ligand (ESL) and P-selectin glycoprotein ligand-1 (PSGL-1) expressed on the surface of neutrophils, monocytes and lymphocyte subsets (Hidalgo et al. 2007). Interaction between endothelial cell selectins and their leukocyte ligands is typically of low affinity yet sufficient to cause rolling of leukocytes on the endothelial surface. Close contact between endothelial cells and leukocytes facilitates stimulation by activated endothelial cell and macrophage-derived chemokines, including interleukin-8 (CXCL8), which recognizes specific G-protein coupled receptors (GPCRs) on neutrophils and monocytes. Triggering by IL- 8 induces neutrophil activation, one consequence of which is a conformational change in $\beta 2$ integrins, principally LFA-1, that allows high affinity interaction with ICAM-1 expressed on the surface of activated endothelial cells. The LFA-1/ICAM-1 interaction causes leukocyte arrest on the endothelium, a step that precedes and is required for diapedesis. The arrested leukocytes can then extravasate and migrate toward the site of tissue injury. Within the tissues, activated neutrophils can phagocytose and destroy harmful agents such as bacteria, thereby playing a major role in clearing the etiology of the injury. Within hours to days, the local chemokine and activated endothelium adhesion receptor repertoire become adapted to help recruit other leukocyte subsets. Thus, the initial wave of neutrophils is followed by the influx of a variety of lymphocyte subsets and monocytes that can differentiate locally into macrophages.

Infiltrating leukocytes release their own panel of cytokines and chemokines, including IL-1, IL-2, IL-4, IL-5, IL10, IL-12, gamma interferon, CCL2, CCL20, CXCL1, CXCL2, CXCL5 and many others, the combination of which helps define the type of inflammatory infiltrate and the corresponding immune reaction. They also release a variety of lipid mediators and growth factors, including VEGF, PDGF, transforming growth factor $\beta(\mathrm{TGF} \beta)$ that stimulate angiogenesis and activate fibroblasts to synthesize and secrete ECM components, as well as numerous proteolytic enzymes, including cathepsins and matrix metalloproteinases (MMPs) that participate in ECM protein breakdown. As the inflammation progresses, and the etiologic agents are gradually eliminated, the balance shifts from inflammation-driven tissue remodelling to tissue repair. A key factor in this transition is the switch in cellderived lipid mediators from the pro-inflammatory prostaglandins to the anti-inflammatory lipoxins. Lipoxins help recruit monocytes rather than neutrophils, which differentiate into macrophages that remove local debris and coordinate subsequent repair. During the early phase of this transition, the area undergoing remodeling contains abundant, highly permeable capillaries, fibroblasts and macrophages and is often referred to as granulation tissue. Granulation tissue is gradually replaced by a provisional matrix, composed principally of collagen III and finally by collagen I that will constitute the scar tissue whose extent depends on the severity of the initial injury, its duration, the regenerative capacity of the host tissue and a variety of as yet poorly understood individual traits.

If the acute inflammatory response fails to eliminate the cause of the injury, the inflammatory process may persist but acquires new features. The neutrophil infiltrate is replaced by monocytes and macrophages, and depending on the nature of the pathogen, by lymphocytes. If the effect of these cells is still insufficient to clear the tissue of the offending agent, a chronic inflammatory state develops. Depending on the pathogen and type of host response, the inflammation may be granulomatous or lymphocytic with the formation of tertiary lymphoid tissues.

\section{Inflammation and cancer}

In 1863, R. Virchow recorded the presence of leukocytes in neoplastic lesions and hypothesized that malignant transformation originates from chronically inflamed tissues (Balkwill and Mantovani 2001), providing the first conceptual framework for linking inflammation and cancer. Clinical and experimental observations of the past two decades increasingly support the notion that immune cells recruited to and activated within the tumor microenvironment play a strong supporting role in tumor progression (Brigati et al. 2002). The release of a myriad of inflammatory mediators including cytokines, chemokines, growth factors, proteases, lipids and other molecules, by the combination of tumor and stromal cells, have been shown to modulate the tumor 
environment in order to regulate the adhesion, invasion and motility of the cells, by the way of autocrine and paracrine signalling pathways (Balkwill 2004).

\section{Recruitment of leukocytes by tumors}

The inflammatory response to tumor growth may be induced by at least two non-mutually exclusive mechanisms. Tumor growth and invasion are associated with significant cell death, some of which occurs by apoptosis and some of which occurs by necrosis. Necrotic cells release numerous mediators of inflammation, including the high mobility group box 1 protein (HMGB1) which is a potent stimulator of macrophages (Chen et al. 2007). Similar to normal cells dying as a result of trauma or infection, tumor cells undergoing necrosis may trigger an analogous tissue response. The second mechanism whereby tumor cells may induce an inflammatory response is by secreting cytokines and chemokines that activate resident fibroblasts, macrophages and mast cells and recruit monocytes and lymphocytes. A key coordinator of innate immunity and inflammation that also behaves as an endogenous tumor promoter in NF- $\kappa \mathrm{B}$ (Maeda and Omata 2008). In leukocytes, NF- $\kappa \mathrm{B}$ functions downstream of pathogen or tissue injury sensors, including the Toll-like receptor (TLR)-MyD88, and the IL-1 receptor (IL-1R) and TNF $\alpha$ receptor (TNFR) signalling pathways (Naugler and Karin 2008). In tumor cells, NF- $\kappa \mathrm{B}$ can be activated as a result of cell autonomous genetic alterations. Its action in both normal and tumor cells consists of inducing the expression of inflammatory cytokines, adhesion receptors, angiogenic factors, inducible nitric oxide synthase (iNOS) and enzymes implicated in the synthesis of arachidonic acid metabolites, including cyclooxygenase-2 (COX-2). NF- $\kappa \mathrm{B}$ is also a major survival factor by virtue of its ability to induce expression of $\mathrm{Bcl} 2$.

Examination of most primary invasive as well as metastatic tumor biopsies reveals an inflammatory infiltrate that varies in composition and intensity (Fig. 2). Infiltrates may range from sparse myeloid cells and $\mathrm{T}$ cells to dense populations of neutrophils, monocytes/macrophages, dendritic cells, natural killer cells or $\mathrm{T}$ and $\mathrm{B}$ lymphocytes (Brigati et al. 2002). The functional role of the various leukocyte components of any tumor infiltrate remains to be fully elucidated, but evidence suggests that it may differ from that observed in the context of physiological tissue repair. Thus, $\mathrm{T}$ cell infiltration of the neoplastic microenvironment may be expected to be beneficial for cancer patient (Wakabayashi et al. 2003; Leffers et al. 2008), yet it often fails to correlate with good prognosis. Abundance of innate immune cells, including mast cells and macrophages, often correlates with angiogenesis and poor prognosis (Nonomura et al. 2007; Taskinen et al. 2008). Current opinion, based on several lines of experimental evidence, holds that inflammation can be both cancer stimulatory and cancer inhibitory, depending on the composition of the infiltrating cell populations, the context, and stage of tumor development. Precise evaluation of the numbers of macrophages and $\mathrm{T}$ cells within cancer nests compared to the stroma has been suggested as a means to obtain a biomarker for
Fig. 2 Variability of the inflammatory response in different carcinomas. a Lung adenocarcinoma, $\mathbf{b}$ pancreatic adenocarcinoma, $\mathbf{c}$ invasive ductal breast carcinoima, $\mathbf{d}$ invasive ductal breast carcinoma with abundant collagen deposition but few inflammatory cells. Arrows indicate inflammatory infiltrates

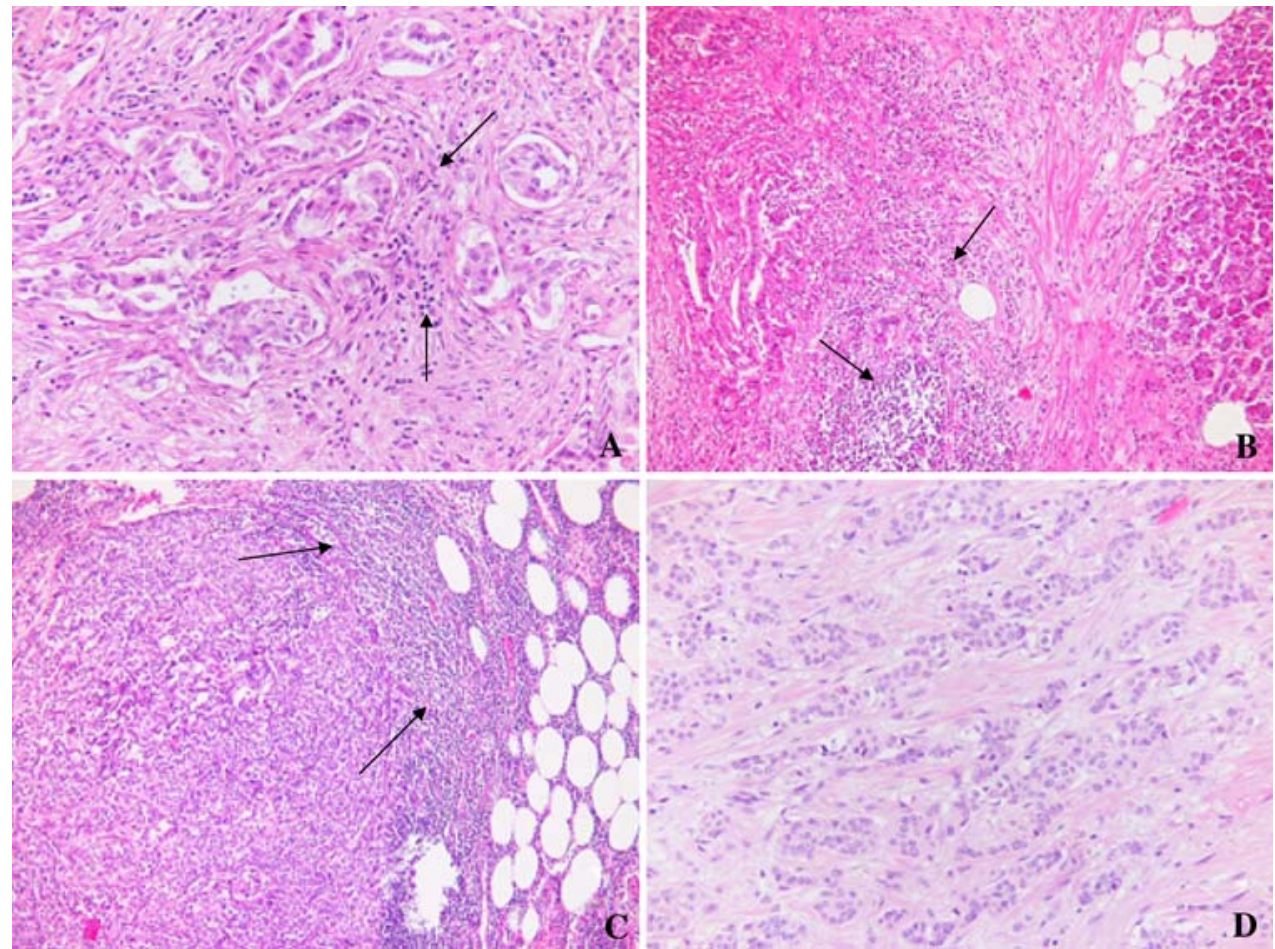


predicting the prognosis of certain types of cancer (Kawai et al. 2008).

\section{The role of macrophages and lymphocytes in tumor growth}

Macrophages are often the most abundant immune cell population in the tumor microenvironment. Recruitment of monocyte precursors circulating in the blood leads to their differentiation into tumor-associated macrophages (TAM). The CCL2 chemokine released by tumor cells, may be a key TAM chemoattractant (Matsushima et al. 1989) along with other molecules including, VEGF, PDGF, TGF $\beta$ and macrophage colony stimulating factor (M-CSF) (Coussens and Werb 2002).

Just as microenvironmental cytokines selectively promote a Th1 or Th2 immune response, they may also polarize macrophages toward the M1 or M2 phenotype, each expressing specialized functional properties. Interferon- $\gamma$ $(\mathrm{IFN} \gamma)$ and microbial molecules induce activation of M1 macrophages, which can support a Th1 immune response, while IL-4, IL-10 and IL-13 induce M2 macrophage activation and promote a Th2 response (Mantovani et al. 2004). The phenotype of TAM resembles that of M2 cells, with pro-tumor functions that include inhibition of Th1 adaptive immunity, production of growth and survival factors including, epidermal growth factor (EGF), IL-6 and CXCL8, and the secretion of angiogenic factors including, VEGF, PDGF, TGF $\beta$, along with a variety of chemokines. TAM can also induce the degradation and remodelling of ECM via the expression of MMPs, and suppress Th1 adaptive immune responses by the production of immunosuppressive mediators (IL-10, PGE2, TGF $\beta$ ) and chemokines capable of recruiting Th2 cells (CCL17, CCL18 and CCL22) (Mantovani et al. 2002; Allavena et al. 2008b). Taken together, TAM play an important role in supporting tumor progression, tissue remodeling, and angiogenesis, while suppressing Th1 type adaptive immunity (Sica and Bronte 2007).

Recent evidence suggests that in some tumor models, TAM are an obligate tumor cell partner during intravasation and initiation of metastasis (Bunt et al. 2007).

Newly formed vessels not only supply tumor cells with nutrients, but provide them with a means to disseminate to other organs. Deletion of colony stimulating factor-1 (CSF1) in a mouse model of multistage mammary carcinogenesis revealed reduced angiogenic response, diminished tumor invasion and abrogation of lung metastasis as a result of deficient macrophage recruitment into the tumor microenvironment (Lin et al. 2001). Intravital imaging of murine mammary carcinoma models provides direct evidence that macrophages enhance metastasis by stimulating tumor motility and facilitating tumor cell intra- and extravasation (Wyckoff et al. 2004). Consistent with these observations, the number of macrophages present in the tumor stroma correlates with increased microvessel density, cell proliferation and tumor size in breast cancers and decreased survival in endometrial cancers (Tsutsui et al. 2005). In other experiments, depletion of peritoneal macrophages resulted in slower tumor development from tumor cells injected into the peritoneal cavity or portal vein (Lin and Pollard 2007). All of these observations suggest that macrophage infiltrates potentiate tumor cell growth and dissemination, at least in part by promoting angiogenesis. Other leukocytes, including mast cells and neutrophils that infiltrate the neoplastic stroma can contribute to angiogenesis by producing many of the angiogeneic factors secreted by macrophages and by releasing proteolytic enzymes that degrade the ECM and liberate sequestered growth factors (Ribatti et al. 2004).

Lymphocytes may play a dual role in tumor progression. On the one hand tumor-specific cytotoxic $\mathrm{T}$ cells have the capacity the eliminate tumor cells by direct or antibodydependent target cell killing. However, recruitment of regulatory T cells and secretion of IL-10 and TGF $\beta$ by either the tumor cells or TAM blunts the immune response and may neutralize the cytolytic potential of cytotoxic $\mathrm{T}$ cells (Mantovani et al. 2008). Having produced or harnessed an effective protection mechanism against immune attack, tumor cells may draw benefits from lymphoid cell infiltration. Different lymphocyte subsets secrete IL-2, IL-3, IL-4, IL-5, IL-10, IL-12, IL-13 and TGF $\beta$ that can help amplify the inflammatory response on the one hand and directly affect tumor cells on the other.

Consistent with the M2 phenotype of TAM, pre-malignant and malignant tissues are reported to be associated with enhanced humoral immunity at the expense of Th1 cell and cytoxic T lymphocyte activity, both of which are suppressed (Agarwal et al. 2006; Johansson et al. 2008). By enhancing humoral immunity and suppressing cytotoxic $\mathrm{T}$ cell activity, Th2 cell-derived IL4 and IL-10 increase the risk of neoplastic transformation in tissues afflicted with chronic inflammatory disease and that harbor initiated neoplastic cells (Tan and Coussens 2007).

\section{Inflammatory mediators in the tumor microenvironment}

Amplification of the inflammatory response consists of an increased influx of leukocytes into the tumor site that results in the secretion and continuous activation of mediators of inflammation, including cytokines, chemokines, cell-derived arachidonic acid metabolites, nitric oxide and reactive oxygen species (ROS), proteolytic enzymes and plasma-derived coagulation factors and complement. The 
combined activity of these mediators is instrumental in influencing and regulating cell proliferation, migration, angiogenesis, tissue remodelling, metabolism and genomic integrity (Hussain and Harris 2007).

\section{Pro-inflammatory cytokines}

The cytokine repertoire produced in the initial response to tissue injury plays a major role in determining the nature of the cellular infiltrate and the corresponding immune response. The pro-inflammatory cytokines IL- 1 and TNF $\alpha$ are induced by hypoxia, which is a common feature of tumor but not of healthy tissues under physiological conditions (Koong et al. 2000). Both cytokines stimulate their own and each other's production, thereby establishing an important amplification loop of the local inflammatory response (Apte and Voronov 2008).

TNF $\alpha$ can be detected in malignant and/or stromal cells in several human carcinomas, lymphomas, and leukaemias, often in association with IL-1. Although TNF $\alpha$ is involved in the homeostasis of the immune system, inflammation and host defence, it can also display pro-tumorigenic effects. Thus, mice lacking the gene that encodes $\mathrm{TNF} \alpha$ are resistant to skin carcinogenesis initiated by dimethylbenzanthracene (DMBA) and promoted by phorbolesters, underscoring the importance of $\mathrm{TNF} \alpha$ during the early stages of tumor development (Moore et al. 1999). Consistent with this notion, overexpression of the TNF $\alpha$ gene can also confer a metastatic phenotype on primary tumor cells (Malik et al. 1990). Several of the known consequences of $\mathrm{TNF} \alpha$ production by cancer cells that help explain its role in cancer progression, have been recently reviewed (Balkwill 2006).

The IL- 1 family includes IL- $1 \alpha$, IL- $1 \beta$, IL- 1 receptor antagonist (IL-1Ra), IL-18 and IL-33 (reviewed by Arend et al. 2008). IL-1 $\beta$, IL-18 and IL-33 are synthesized as precursors and must be processed by the cysteine protease caspase- 1 to biologically active mature forms that are released from cells. Depending on the stimulus, caspase-1, also called IL-1 $\beta$ converting enzyme (ICE), can induce secretion of pro-inflammatory cytokines or trigger cell pyroptosis, a caspase-1-dependent cell death pathway (Ting et al. 2008). Pyroptosis is characterized by loss of plasma membrane integrity, resulting in the release of pro-inflammatory cellular contents into the microenvironment that induce and maintain the inflammatory process (Fink and Cookson 2005). Caspase-1 itself requires cleavage by a multiprotein complex to become active and process IL- $1 \beta$. The multiprotein complex, called the inflammasome, is composed of several distinct proteins including the Nodlike receptor protein Nalp3. The Nalp3 inflammasome is assembled in response to a variety of signals, including the presence of asbestos, ROS and monosodium urate, and trig- gers robust secretion of IL- $1 \beta$ that may lead to a chronic inflammatory state in the lung (for the case of asbestos), joints (monosodium urate, gout) or other organs (Petrilli and Martinon 2007; Dostert et al. 2008).

The IL-1 family induces expression of numerous proinflammatory genes, including COX-2, the inducible NOS, MMPs and pro-inflammatory cytokines capable of recruiting leukocytes to the tumor site. Endothelial cell proliferation has been shown to be stimulated by IL-1, as has expression of integrins and other adhesion receptors, all of which constitute mechanisms whereby IL-1 family members promote the infiltration of inflammatory cells, tumor invasiveness and angiogenesis (Voronov et al. 2003; Apte and Voronov 2008). This notion has been corroborated using IL-1Ra, which binds the IL-1 receptor but has no biological activity and thereby constitutes a competitive inhibitor of IL-1 $\beta$. Delivery of IL-1Ra to the tumor site has been observed to reduce local inflammation, inhibit cancer progression and decrease lung metastases in mice at least in part by counteracting the proangiogenic effect of IL- $1 \beta$, (Bar et al. 2004; Lavi et al. 2007). These observations raise the possibility that IL-1Ra may constitute an attractive reagent in anticancer therapy, in addition to its already promising effects in chronic inflammatory diseases.

Although experimental models provide and essential vehicle toward elucidating the effects of any given cytokine on tumorigenesis and tumor progression, they often provide an oversimplification of the actual physiological role within the natural context of the tumor. Cytokines in fact occasionally display dual roles and dose-dependence in the physiological context of a tumor, rendering true assessment of their function challenging. For example, in addition to its central role in inflammation, the proform of IL- $1 \alpha$ can have an oncogenic effect when overexpressed in mesangial cells (Stevenson et al. 1997). Similarly, the dual effect of IL-18 in tumor progression is illustrated by its ability to stimulate natural killer and $\mathrm{T}$ cells which eliminate nascent tumor cells on the one hand, yet to display pro-carcinogenic functions on the other, that include promotion of VEGF production, which itself stimulates IL-18 mRNA synthesis in a dose-dependent manner. (Kim et al. 2007). IL-18 also induces MMP-9 expression, and may thereby be a significant player in tumor cell migration, invasion and metastasis (Park et al. 2007).

\section{Reactive oxygen and nitrogen species (ROS and RNS)}

ROS are generated by normal physiological processes, including aerobic metabolism and inflammatory responses. At physiological concentrations, they participate in the regulation of a variety of cellular processes ranging from transcription to proliferation. In specialized cells, such as neutrophils, they can be generated in bursts within defined 
cellular compartments that result in efficient microbial killing. In excess (oxidative stress), they can inflict cellular damage and play an important role in tumorigenesis.

ROS and reactive nitrogen species (RNS) are widely released in tumor microenvironment by activated immune cells and can function as chemical effectors in inflammation-driven carcinogenesis. ROS and RNS can cause DNA damage that results in replication errors and genomic instability, contributing to tumor initiation, and RNS can also inactivate DNA repair enzymes (Jaiswal et al. 2000; Marnett 2000). Excess of nitric oxide has been shown to induce expression of a variety of transcription factors associated with the regulation of tumor growth (AP-1, p53) and to modulate their activity or stability or those of their inhibitors (NF- $\kappa \mathrm{B}$ ). In addition, RNS can modulate accessibility of promoters via increased DNA methylation or histone deacetylation (Kroncke 2003).

Nitric oxide can enhance migration, invasion and metastasis of breast and colon cancer cells by activating the MAPK pathway (Jadeski et al. 2003). It also increases VEGF expression in tumor cells, promoting angiogenesis and tumor progression, and induces posttranslational modification of key regulators of tumor growth and inflammation, including p53and COX-2 (Fukumura et al. 2006). Nitric oxide distribution and localization are critical for its effect on tumor propagation: its concentration has been shown to be increased in stromal fibroblasts and immune cells in many human tumors, independent of its production by tumor cells. Host produced nitric oxide has been suggested to have tumoricidal activity and to participate in the suppression of tumor growth and metastasis in mice (Wei et al. 2003).

\section{Growth factors}

Beyond a critical size of about $400 \mu$, that may vary according to tumor type, the initial aggregate of tumor cells requires its own blood supply. This is typically achieved by the induction of angiogenesis as a result of synthesis and secretion of angiogenic factors that include VEGF, bFGF, TGF $\beta$, angiopoietin-2 and several others (Karamysheva 2008). Whereas some tumor cells are capable of secreting their own angiogenic factors, others rely on host cell-driven angiogenesis. In ovarian and breast cancer, leukocytes, and particularly TAM secrete abundant VEGF as well as a variety of proteolytic enzymes, including urokinase-type plasminogen activator (uPA) and MMP-9 that participate in angiogenesis.

Arachidonic acid metabolites

The two mains classes of arachidonic acid derivatives are prostaglandins and leukotrienes, both of which participate actively in the regulation of inflammation.
Prostaglandins are cyclooxygenase-dependent derivatives of arachidonic acid that display proinflammatory activity ranging from the regulation of vascular volume and permeability to platelet aggregation. A strong argument that inflammation supports tumor growth and progression stems from the observation that non-steroidal anti-inflammatory drugs that block COX-2 have a potent inhibitory effect on tumor growth (Gupta and Dubois 2001; Rostom et al. 2007). COX-2 is an inducible isoenzyme that acts as an immediate early gene expressed in response to cytokines, growth factors, and other stimuli to produce prostaglandins. Because COX-2 is usually overexpressed in colon cancer, COX-2 inhibitors are currently being tested as a therapeutic option in colon cancer management. However, despite promising results in colon cancer prevention and a tangible reduction in the incidence of colon adenomas, nonsteroidal anti-inflammatory drugs and COX-2 inhibitors can incur severe cardiovascular and gastrointestinal side effects that hinder their routine use for cancer treatment (Bertagnolli et al. 2006; Rostom et al. 2007).

\section{Proteolytic enzymes}

Enzymes released by leukocytes also contribute to the continuous tissue remodelling that characterizes cancer. Macrophages, as well as neutrophils and lymphocytes express and secrete a variety of MMPs that together have the potential to hydrolyse all known ECM proteins (for review, see Van Kempen et al. 2006). Continued release and activation of MMPs results in degradation of ECM glycoproteins and proteoglycans with several major consequences. First, degradation of macromolecules such as collagens reduces the physical barriers that tumor cells have to overcome as they migrate toward lymphatic and blood vessels. Second, some of the ECM protein degradation products, display bioactivity of their own. A prominent example is the release of cryptic fragments of laminin as a result of MMP-mediated cleavage of laminin-5 $\gamma 2$ chains that mimic EGF receptor (EGFR) ligands and induce cell migration and invasive potential in EGFR expressing cells (Schenk et al. 2003). Third, degradation of proteoglycans results in the release and activation of a multitude of ECM-sequestered growth factors, augmenting their bioavailability to tumor cells. Proteolytic activation of growth factor precursors can also occur at the cell surface. Thus, leukocyte-derived MMP-7 cleaves and activates cell surface pro-heparin-binding EGF (HB-EGF) into its bioactive form, which enhances tumor cell migration and survival ( $\mathrm{Li}$ et al. 2006). Together with cathepsins, MMP-7 can also cleave E-cadherin, facilitating tumor cell disaggregation and enhancing motility (Lee et al. 2007). Thus, leukocyte-derived proteolytic enzymes have the ability to alter tumor cell adhesion while promoting migration, survival and growth, and it comes as no surprise 
that increased expression of MMPs in human malignant tissue often correlates with poor prognosis (Egeblad and Werb 2002). One important aspect of leukocyte-derived MMPs is that they may provide the tissue remodelling force that certain malignant cells may not have, since not all tumor types display high expression levels of MMPs and other proteolytic enzymes. By recruiting and harnessing the inflammatory response, weakly invasive tumor cells may acquire the means to disseminate.

\section{Systemic mediators}

Inflammation enhances coagulation, both by augmenting platelet aggregation and by inducing regulators of the clotting cascade, including plasmin and uPA. Circulating tumor cells can express cell surface receptors decorated by oligosaccharides that constitute selectin ligands (Borsig et al. 2001). In activated platelets, preformed P-selectin sequestered within $\alpha$-granules is translocated to the cell surface and mediates platelet binding to tumor cells expressing the corresponding ligands. Similarly, leukocytes expressing Lselectin can bind to circulating tumor cells leading to the formation of large aggregates that may create microemboli in the capillaries and facilitate arrest within the vasculature of distant organs. Such aggregates may also serve to protect tumor cells from shear stress in the circulation and aggression by natural killer cells and cytotoxic lymphocytes (Barthel et al. 2007).

Plasmin and uPA participate in ECM and fibrin degradation that contribute to tissue remodelling and facilitate tumor cell invasion.

Similar to inflammation induced by bacteria or trauma, complement activation constitutes an integral part of cancer-associated inflammation. Sustained complement activation results in continuous stimulation of macrophages, mast cells and neutrophils. Complement fragments, particularly C5a are also among the most potent chemotactic molecules for a variety of leukocytes and ensure continued recruitment of leukocytes to sites of tumor growth.

\section{Preparation of metastatic niches}

Following injection of B16F1 melanoma cells into mice, only $2 \%$ are observed to form micrometastatic foci, and less than $0.2 \%$ activate angiogenesis and develop a secondary tumor (Luzzi et al. 1998). At first glance, these "selected" cells capable of forming metastases seems to be rare. However, proliferation of these cells at secondary sites may not be a reflection of their intrinsic properties, but rather that of the preparation of the local tissue or "soil" to provide them with a permissive microenvironment. Recent evidence suggests that hematopoietic progenitor cells first home to pre-metastatic sites and promote recruitment of tumor cells from the blood, favoring their subsequent outgrowth by the robust expression of VEGF receptor (Kaplan et al. 2005). Consistent with this notion, the release of VEGF, TGF $\beta$ and TNF $\alpha$ from the primary tumor site was shown to promote the expression of S100 proteins, which act as chemoattractants for tumor cells in premetastatic lung sites (Hiratsuka et al. 2006). Because TAM and mast cells are potent producers of TNF $\alpha, \operatorname{TGF} \beta$ and VEGF, the inflammatory infiltrates at primary tumor sites may be implicated in the formation of the premetastatic niche by releasing these pro-inflammatory factors into the circulation. If the chemoattracted tumor cells encounter hematopoietic progenitor cells at such premetastatic sites, the angiogenic switch necessary for subsequent metastatic cell survival and outgrowth could be facilitated (for review, see DeNardo et al. 2008).

\section{Chronic inflammation as a predisposition to cancer}

Substantial evidence supports the notion that inflammation participates in providing the conditions that lead to transformation. Colon cancer, hepatocellular carcinoma, esophageal and gastric cancer, Epstein-Barr virus-associated Burkitt's lymphoma and human papilloma virus (HPV)associated cervical cancer are all salient examples of tumors arising in a context of chronic inflammation.

Common inducers of chronic inflammatory responses include viral and bacterial infections as well as prolonged exposure to irradiation or environmental carcinogens. These infections and chronic inflammation contribute to at least $25 \%$ of all cancer cases. The multifactorial network of inflammatory mediators, including ROS, imposes a stress that can induce genetic and epigenetic changes as well as relevant post-translational modifications of macromolecules. In the medium to long term, sustained inflammatory stress can cause alterations in critical signalling pathways responsible for maintaining cellular homeostasis, leading to growth dysregulation and cancer (Hussain and Harris 2007).

The association between Crohn's disease, and particularly ulcerative colitis, and colon cancer is well established (Eaden et al. 2000). Similarly, there is a tight association between hepatitis $\mathrm{B}(\mathrm{HBV})$ and $\mathrm{C}(\mathrm{HCV})$ viruses and hepatocellular carcinoma (Matsuzaki et al. 2007). In both cases of hepatitis, the mechanism whereby viral genes induce transformation is unclear. The HBx gene of the HBV genome has been implicated in transformation, presumably by affecting both p53 and Rb (Kwun and Jang 2004). However, hepatocellular carcinoma can also arise in the context of alcoholic liver cirrhosis that is unrelated to viral infection. It is believed that the chronic 
inflammation induced by $\mathrm{HBV}$ and $\mathrm{HCV}$ plays a role in stimulating hepatocyte proliferation and augments the likelihood of developing transforming mutations. Cirrhosis represents a more obvious terrain for cancer development, where chronic inflammation leads to aberrant liver regeneration that presents an increased risk for oncogenic mutations to occur.

Helicobacter pylori, a gram-negative bacterium associated with gastric ulcer, is an established carcinogen in gastric cancer development, the second most frequent malignancy in the world. Chronic inflammation that accompanies ulcers is believed to be the condition that causes DNA damage leading to malignant transformation of gastric epithelial cells (Lochhead and El-Omar 2007; McNamara and El-Omar 2008).

Recent work has shed light onto the molecular pathways that link gastrointestinal tumorigenesis and inflammation. Mutation of the gene encoding the APC protein, frequently associated with gastrointestinal malignancies, induces chronic activation of the $\mathrm{Wnt} / \beta$-catenin signalling pathway, which plays a central role in modulating the balance between stemness and differentiation in several cell types (Fodde and Brabletz 2007). Under pressure of $\mathrm{H}$. pylori, macrophages are continuously recruited by the sustained presence of pro-inflammatory cytokines. Hence, the abundantly available macrophage-derived TNF $\alpha$ engages its receptor on gastric epithelial cells leading to nuclear accumulation of $\beta$-catenin, which promotes growth dysregulation initiated by the APC mutation (Oguma et al. 2008).

HPV and EBV-associated malignancies are typical examples of tumors arising the in the context of viral infections. Although HPV and EBV-mediated transformation is supported by well established mechanistic events within the infected cells themselves, the resulting inflammatory response and suppression of adaptive immunity are both believed to play a major role in the subsequent emergence of malignant tumors. In a mouse model of cancer induce by HPV, antibodies have been shown to be deposited in the stroma. By binding to ECM molecules, these antibodies trigger an inflammatory response than favors tumor progression (Psyrri and DiMaio 2008).

Although inflammation alone has not yet been shown to be a sufficient condition for cancer development, recent work further strengthens the link between these two events. Using $\mathrm{Nalp}^{-1-}$ mice, investigators have shown diminished recruitment of inflammatory cells to the lungs in response to asbestos inhalation, with lower corresponding cytokine production (Dostert et al. 2008). These observations place the Nalp3 inflammasome at the center of asbestos-induced chronic lung inflammation that is well known to promote transformation and malignant tumor development.

\section{Anti-tumor inflammation}

Crosstalk between innate and adaptive immune responses is mediated by complex interactions between the various cellular players, and it seems conceptually reasonable that the vast array of soluble factors produced within the tumor microenvironment can lead to dysregulation of the immune system, which often favors tumor growth. During acute inflammatory reactions, the innate immune system activates and regulates the adaptive immunity. However, in the case of chronic tissue inflammation, it is often the opposite scenario that is observed, where excessive activation of the innate immune response, mediated by a dysregulated adaptive immune system, culminates in tissue damage (De Visser and Coussens 2005). Thus, innate and adaptive immunity both have the potential to promote or delay tumor onset and progression, depending on the balance between the pro- and anti-tumor mediators synthesized by the various immune cells.

Despite the accumulating clinical and experimental evidence that inflammation has tumor-stimulating activity, there are several examples where the opposite may hold true. Some chronic inflammatory states, a salient example being psoriasis, are not associated with an increased risk of developing cancer. In addition, the presence of inflammatory cells can be an indicator of favorable prognosis in some tumor types (for example the presence of eosinophils in colon cancer and TAM in pancreatic carcinoma). These observations suggest that at least in some situations, inflammatory cells may be able to eliminate tumor cells just as they can destroy normal cell counterparts. Experimental evidence supports this notion based on the observation that appropriately activated macrophages can kill tumor cells and elicit a tumor-inhibitory inflammatory response (Allavena et al. 2008a). A key player in determining the type of macrophage response to tumor cells appears to be NF- $\kappa \mathrm{B}$ (Hayden et al. 2006). NF- $\kappa \mathrm{B}$ may therefore constitute a therapeutic target that could potentially be re-programmed to direct macrophages to exert their anti-tumor properties.

Lessons from psoriasis may be important in understanding what regulates the balance between pro- and anti-tumor inflammatory responses. Psoriasis is a Th1 cell-mediated disorder characterized by massive infiltration of neutrophils and monocytes in the skin (Nickoloff et al. 2005). The monocytes can differentiate into macrophages with an M1 phenotype, which display anti-tumor activity. Hence, the inflammatory response associated with psoriasis does not facilitate tumor development because of the overwhelming presence of macrophages capable of eliminating nascent tumor cells. It should be borne in mind that potential duality of the inflammatory response to cancer can be regulated by the tissue type, as suggested by a skin tumor model 
where overexpression of NF- $\kappa \mathrm{B}$ was observed to inhibit invasive epidermal carcinoma (Dajee et al. 2003).

The precise mechanisms that help trigger an anti-cancer inflammatory response remain to be elucidated. However, it would seem that being able to steer inflammation toward a Th1/M1 versus Th2/M2 type response may provide a powerful means to control tumor growth. How to achieve this will obviously require a better understanding of the pro-and anti-tumor microenvironments and the molecular mechanisms that guide their constitution.

\section{Perspectives}

Despite an abundance of new insight into the association between inflammation and cancer development and progression, numerous important questions remain. It has still not been clearly established whether chronic inflammation can in and of itself, in the absence of an exogenous carcinogen, lead to transformation. Although there are indications that inflammation can cause cancer, formal proof is still lacking.

Whereas most malignant tumors are associated with inflammatory infiltrates, it would seem that the composition of the infiltrates varies from tumor to tumor and even according to the stage of tumor development. What regulates the type of inflammatory response to any given tumor type and are there functionally relevant and therapeutically targetable common denominators among inflammatory responses to various tumor types? Answers to these questions will be crucial.

There is strong evidence that Th1/M1 responses may protect from tumor development whereas Th2/M2 responses support tumor development and progression. What are the microenvironmental conditions that determine which type of response occurs, and how might we be able to re-direct Th2/M2 to Th2/M1 type responses?

Clearly, we need a much better understanding of cancerassociated inflammation in order to target it in patients with cancer. However, the abundance of recent discoveries predicts that cancer-related inflammation research is poised for exciting times.

Open Access This article is distributed under the terms of the Creative Commons Attribution Noncommercial License which permits any noncommercial use, distribution, and reproduction in any medium, provided the original author(s) and source are credited.

\section{References}

Agarwal A, Verma S, Burra U, Murthy NS, Mohanty NK, Saxena S (2006) Flow cytometric analysis of Th1 and Th2 cytokines in PBMCs as a parameter of immunological dysfunction in patients of superficial transitional cell carcinoma of bladder. Cancer Immunol Immunother 55:734-743
Allavena P, Sica A, Garlanda C, Mantovani A (2008a) The Yin-Yang of tumor-associated macrophages in neoplastic progression and immune surveillance. Immunol Rev 222:155-161

Allavena P, Sica A, Solinas G, Porta C, Mantovani A (2008b) The inflammatory micro-environment in tumor progression: the role of tumor-associated macrophages. Crit Rev Oncol Hematol 66:1-9

Apte RN, Voronov E (2008) Is interleukin-1 a good or bad 'guy' in tumor immunobiology and immunotherapy? Immunol Rev 222:222-241

Arend WP, Palmer G, Gabay C (2008) IL-1, IL-18, and IL-33 families of cytokines. Immunol Rev 223:20-38

Balkwill F (2004) Cancer and the chemokine network. Nat Rev Cancer 4:540-550

Balkwill F (2006) TNF-alpha in promotion and progression of cancer. Cancer Metastasis Rev 25:409-416

Balkwill F, Mantovani A (2001) Inflammation and cancer: back to Virchow? Lancet 357:539-545

Bar D, Apte RN, Voronov E, Dinarello CA, Cohen S (2004) A continuous delivery system of IL-1 receptor antagonist reduces angiogenesis and inhibits tumor development. FASEB J 18:161-163

Barthel SR, Gavino JD, Descheny L, Dimitroff CJ (2007) Targeting selectins and selectin ligands in inflammation and cancer. Expert Opin Ther Targets 11:1473-1491

Bertagnolli MM, Eagle CJ, Zauber AG, Redston M, Solomon SD, Kim K, Tang J, Rosenstein RB, Wittes J, Corle D, Hess TM, Woloj GM, Boisserie F, Anderson WF, Viner JL, Bagheri D, Burn J, Chung DC, Dewar T, Foley TR, Hoffman N, Macrae F, Pruitt RE, Saltzman JR, Salzberg B, Sylwestrowicz T, Gordon GB, Hawk ET (2006) Celecoxib for the prevention of sporadic colorectal adenomas. N Engl J Med 355:873-884

Borsig L, Wong R, Feramisco J, Nadeau DR, Varki NM, Varki A (2001) Heparin and cancer revisited: mechanistic connections involving platelets, P-selectin, carcinoma mucins, and tumor metastasis. Proc Natl Acad Sci USA 98:3352-3357

Brigati C, Noonan DM, Albini A, Benelli R, Ben-Baruch A (2002) Tumors and inflammatory infiltrates: friends or foes? Inflammationassociated immune suppression in cancer: the roles played by cytokines, chemokines and additional mediators. Clin Exp Metastasis 19:247-258

Bunt SK, Yang L, Sinha P, Clements VK, Leips J, Ostrand-Rosenberg $S$ (2007) Reduced inflammation in the tumor microenvironment delays the accumulation of myeloid-derived suppressor cells and limits tumor progression. Cancer Res 67:10019-10026

Chen K, Huang J, Gong W, Iribarren P, Dunlop NM, Wang JM (2007) Toll-like receptors in inflammation, infection and cancer. Int Immunopharmacol 7:1271-1285

Coussens LM, Werb Z (2002) Inflammation and cancer. Nature 420:860-867

Dajee M, Lazarov M, Zhang JY, Cai T, Green CL, Russell AJ, Marinkovich MP, Tao S, Lin Q, Kubo Y, Khavari PA (2003) NF-kap$\mathrm{paB}$ blockade and oncogenic Ras trigger invasive human epidermal neoplasia. Nature 421:639-643

De Visser KE, Coussens LM (2005) The interplay between innate and adaptive immunity regulates cancer development. Cancer Immunol Immunother 54:1143-1152

DeNardo DG, Johansson M, Coussens LM (2008) Immune cells as mediators of solid tumor metastasis. Cancer Metastasis Rev 27:11-18

Dostert C, Petrilli V, Van Bruggen R, Steele C, Mossman BT, Tschopp J (2008) Innate immune activation through Nalp3 inflammasome sensing of asbestos and silica. Science 320:674-677

Dvorak HF (1986) Tumors: wounds that do not heal. Similarities between tumor stroma generation and wound healing. N Engl J Med 315:1650-1659

Eaden J, Abrams K, Ekbom A, Jackson E, Mayberry J (2000) Colorectal cancer prevention in ulcerative colitis: a case-control study. Aliment Pharmacol Ther 14:145-153 
Egeblad M, Werb Z (2002) New functions for the matrix metalloproteinases in cancer progression. Nat Rev Cancer 2:161-174

Fink SL, Cookson BT (2005) Apoptosis, pyroptosis, and necrosis: mechanistic description of dead and dying eukaryotic cells. Infect Immun 73:1907-1916

Fodde R, Brabletz T (2007) Wnt/beta-catenin signaling in cancer stemness and malignant behavior. Curr Opin Cell Biol 19:150-158

Fukumura D, Kashiwagi S, Jain RK (2006) The role of nitric oxide in tumour progression. Nat Rev Cancer 6:521-534

Gupta RA, Dubois RN (2001) Colorectal cancer prevention and treatment by inhibition of cyclooxygenase-2. Nat Rev Cancer 1:11-21

Hayden MS, West AP, Ghosh S (2006) NF-kappaB and the immune response. Oncogene 25:6758-6780

Hidalgo A, Peired AJ, Wild MK, Vestweber D, Frenette PS (2007) Complete identification of E-selectin ligands on neutrophils reveals distinct functions of PSGL-1, ESL-1, and CD44. Immunity 26:477-489

Hiratsuka S, Watanabe A, Aburatani H, Maru Y (2006) Tumour-mediated upregulation of chemoattractants and recruitment of myeloid cells predetermines lung metastasis. Nat Cell Biol 8:13691375

Hussain SP, Harris CC (2007) Inflammation and cancer: an ancient link with novel potentials. Int J Cancer 121:2373-2380

Jadeski LC, Chakraborty C, Lala PK (2003) Nitric oxide-mediated promotion of mammary tumour cell migration requires sequential activation of nitric oxide synthase, guanylate cyclase and mitogen-activated protein kinase. Int J Cancer 106:496-504

Jaiswal M, LaRusso NF, Burgart LJ, Gores GJ (2000) Inflammatory cytokines induce DNA damage and inhibit DNA repair in cholangiocarcinoma cells by a nitric oxide-dependent mechanism. Cancer Res 60:184-190

Johansson M, Denardo DG, Coussens LM (2008) Polarized immune responses differentially regulate cancer development. Immunol Rev 222:145-154

Kaminski A, Hahne JC, Haddouti el M, Florin A, Wellmann A, Wernert N (2006) Tumour-stroma interactions between metastatic prostate cancer cells and fibroblasts. Int J Mol Med 18:941-950

Kaplan RN, Riba RD, Zacharoulis S, Bramley AH, Vincent L, Costa C, MacDonald DD, Jin DK, Shido K, Kerns SA, Zhu Z, Hicklin D, Wu Y, Port JL, Altorki N, Port ER, Ruggero D, Shmelkov SV, Jensen KK, Rafii S, Lyden D (2005) VEGFR1-positive haematopoietic bone marrow progenitors initiate the pre-metastatic niche. Nature 438:820-827

Karamysheva AF (2008) Mechanisms of angiogenesis. Biochemistry (Mosc) 73:751-762

Kawai O, Ishii G, Kubota K, Murata Y, Naito Y, Mizuno T, Aokage K, Saijo N, Nishiwaki Y, Gemma A, Kudoh S, Ochiai A (2008) Predominant infiltration of macrophages and CD8(+) T Cells in cancer nests is a significant predictor of survival in stage IV nonsmall cell lung cancer. Cancer 113:1387-1395

Kim KE, Song H, Kim TS, Yoon D, Kim CW, Bang SI, Hur DY, Park $\mathrm{H}$, Cho DH (2007) Interleukin-18 is a critical factor for vascular endothelial growth factor-enhanced migration in human gastric cancer cell lines. Oncogene 26:1468-1476

Koong AC, Denko NC, Hudson KM, Schindler C, Swiersz L, Koch C, Evans S, Ibrahim H, Le QT, Terris DJ, Giaccia AJ (2000) Candidate genes for the hypoxic tumor phenotype. Cancer Res 60:883-887

Kroncke KD (2003) Nitrosative stress and transcription. Biol Chem 384:1365-1377

Kwun HJ, Jang KL (2004) Natural variants of hepatitis B virus X protein have differential effects on the expression of cyclin-dependent kinase inhibitor p21 gene. Nucleic Acids Res 32:2202-2213

Lavi G, Voronov E, Dinarello CA, Apte RN, Cohen S (2007) Sustained delivery of IL-1 Ra from biodegradable microspheres reduces the number of murine B16 melanoma lung metastases. J Control Release 123:123-130

Lee KH, Choi EY, Hyun MS, Jang BI, Kim TN, Kim SW, Song SK, Kim JH, Kim JR (2007) Association of extracellular cleavage of E-cadherin mediated by MMP-7 with HGF-induced in vitro invasion in human stomach cancer cells. Eur Surg Res 39:208-215

Leffers N, Gooden MJ, de Jong RA, Hoogeboom BN, Ten Hoor KA, Hollema H, Boezen HM, van der Zee AG, Daemen T, Nijman HW (2008) Prognostic significance of tumor-infiltrating T-lymphocytes in primary and metastatic lesions of advanced stage ovarian cancer. Cancer Immunol Immunother

Li M, Yamamoto H, Adachi Y, Maruyama Y, Shinomura Y (2006) Role of matrix metalloproteinase-7 (matrilysin) in human cancer invasion, apoptosis, growth, and angiogenesis. Exp Biol Med (Maywood) 231:20-27

Lin EY, Nguyen AV, Russell RG, Pollard JW (2001) Colony-stimulating factor 1 promotes progression of mammary tumors to malignancy. J Exp Med 193:727-740

Lin EY, Pollard JW (2007) Tumor-associated macrophages press the angiogenic switch in breast cancer. Cancer Res 67:5064-5066

Lochhead P, El-Omar EM (2007) Helicobacter pylori infection and gastric cancer. Best Pract Res Clin Gastroenterol 21:281-297

Luzzi KJ, MacDonald IC, Schmidt EE, Kerkvliet N, Morris VL, Chambers AF, Groom AC (1998) Multistep nature of metastatic inefficiency: dormancy of solitary cells after successful extravasation and limited survival of early micrometastases. Am J Pathol 153:865-873

Maeda S, Omata M (2008) Inflammation and cancer: role of nuclear factor-kappaB activation. Cancer Sci 99:836-842

Malik ST, Naylor MS, East N, Oliff A, Balkwill FR (1990) Cells secreting tumour necrosis factor show enhanced metastasis in nude mice. Eur J Cancer 26:1031-1034

Mantovani A, Sozzani S, Locati M, Allavena P, Sica A (2002) Macrophage polarization: tumor-associated macrophages as a paradigm for polarized M2 mononuclear phagocytes. Trends Immunol 23:549-555

Mantovani A, Sica A, Sozzani S, Allavena P, Vecchi A, Locati M (2004) The chemokine system in diverse forms of macrophage activation and polarization. Trends Immunol 25:677-686

Mantovani A, Romero P, Palucka AK, Marincola FM (2008) Tumour immunity: effector response to tumour and role of the microenvironment. Lancet 371:771-783

Marnett LJ (2000) Oxyradicals and DNA damage. Carcinogenesis $21: 361-370$

Matsushima K, Larsen CG, DuBois GC, Oppenheim JJ (1989) Purification and characterization of a novel monocyte chemotactic and activating factor produced by a human myelomonocytic cell line. J Exp Med 169:1485-1490

Matsuzaki K, Murata M, Yoshida K, Sekimoto G, Uemura Y, Sakaida N, Kaibori M, Kamiyama Y, Nishizawa M, Fujisawa J, Okazaki K, Seki T (2007) Chronic inflammation associated with hepatitis $\mathrm{C}$ virus infection perturbs hepatic transforming growth factor beta signaling, promoting cirrhosis and hepatocellular carcinoma. Hepatology 46:48-57

McNamara D, El-Omar E (2008) Helicobacter pylori infection and the pathogenesis of gastric cancer: a paradigm for host-bacterial interactions. Dig Liver Dis 40:504-509

Moore RJ, Owens DM, Stamp G, Arnott C, Burke F, East N, Holdsworth H, Turner L, Rollins B, Pasparakis M, Kollias G, Balkwill F (1999) Mice deficient in tumor necrosis factor-alpha are resistant to skin carcinogenesis. Nat Med 5:828-831

Naugler WE, Karin M (2008) NF-kappaB and cancer-identifying targets and mechanisms. Curr Opin Genet Dev 18:19-26

Nickoloff BJ, Ben-Neriah Y, Pikarsky E (2005) Inflammation and cancer: is the link as simple as we think? J Invest Dermatol 124:x-xiv 
Nonomura N, Takayama H, Nishimura K, Oka D, Nakai Y, Shiba M, Tsujimura A, Nakayama M, Aozasa K, Okuyama A (2007) Decreased number of mast cells infiltrating into needle biopsy specimens leads to a better prognosis of prostate cancer. Br J Cancer 97:952-956

Oguma K, Oshima H, Aoki M, Uchio R, Naka K, Nakamura S, Hirao A, Saya H, Taketo MM, Oshima M (2008) Activated macrophages promote Wnt signalling through tumour necrosis factoralpha in gastric tumour cells. EMBO J 27:1671-1681

Olumi AF, Grossfeld GD, Hayward SW, Carroll PR, Tlsty TD, Cunha GR (1999) Carcinoma-associated fibroblasts direct tumor progression of initiated human prostatic epithelium. Cancer Res 59:5002-5011

Park S, Cheon S, Cho D (2007) The dual effects of interleukin-18 in tumor progression. Cell Mol Immunol 4:329-335

Petrilli V, Martinon F (2007) The inflammasome, autoinflammatory diseases, and gout. Joint Bone Spine 74:571-576

Psyrri A, DiMaio D (2008) Human papillomavirus in cervical and head-and-neck cancer. Nat Clin Pract Oncol 5:24-31

Ribatti D, Crivellato E, Roccaro AM, Ria R, Vacca A (2004) Mast cell contribution to angiogenesis related to tumour progression. Clin Exp Allergy 34:1660-1664

Rostom A, Dube C, Lewin G, Tsertsvadze A, Barrowman N, Code C, Sampson M, Moher D (2007) Nonsteroidal anti-inflammatory drugs and cyclooxygenase- 2 inhibitors for primary prevention of colorectal cancer: a systematic review prepared for the U.S. Preventive Services Task Force. Ann Intern Med 146:376-389

Schenk S, Hintermann E, Bilban M, Koshikawa N, Hojilla C, Khokha R, Quaranta V (2003) Binding to EGF receptor of a laminin-5 EGF-like fragment liberated during MMP-dependent mammary gland involution. J Cell Biol 161:197-209

Sica A, Bronte V (2007) Altered macrophage differentiation and immune dysfunction in tumor development. J Clin Invest 117:1155-1166
Stevenson FT, Turck J, Locksley RM, Lovett DH (1997) The N-terminal propiece of interleukin 1 alpha is a transforming nuclear oncoprotein. Proc Natl Acad Sci USA 94:508-513

Tan TT, Coussens LM (2007) Humoral immunity, inflammation and cancer. Curr Opin Immunol 19:209-216

Taskinen M, Karjalainen-Lindsberg ML, Leppa S (2008) Prognostic influence of tumor-infiltrating mast cells in patients with follicular lymphoma treated with rituximab and CHOP. Blood 111:4664 4667

Ting JP, Willingham SB, Bergstralh DT (2008) NLRs at the intersection of cell death and immunity. Nat Rev Immunol 8:372-379

Tlsty TD, Coussens LM (2006) Tumor stroma and regulation of cancer development. Annu Rev Pathol 1:119-150

Tsutsui S, Yasuda K, Suzuki K, Tahara K, Higashi H, Era S (2005) Macrophage infiltration and its prognostic implications in breast cancer: the relationship with VEGF expression and microvessel density. Oncol Rep 14:425-431

Van Kempen LC, De Visser KE, Coussens LM (2006) Inflammation, proteases and cancer. Eur J Cancer 42:728-734

Voronov E, Shouval DS, Krelin Y, Cagnano E, Benharroch D, Iwakura Y, Dinarello CA, Apte RN (2003) IL-1 is required for tumor invasiveness and angiogenesis. Proc Natl Acad Sci USA 100:2645-2650

Wakabayashi O, Yamazaki K, Oizumi S, Hommura F, Kinoshita I, Ogura S, Dosaka-Akita H, Nishimura M (2003) CD4+ T cells in cancer stroma, not CD8+ T cells in cancer cell nests, are associated with favorable prognosis in human non-small cell lung cancers. Cancer Sci 94:1003-1009

Wei D, Richardson EL, Zhu K, Wang L, Le X, He Y, Huang S, Xie K (2003) Direct demonstration of negative regulation of tumor growth and metastasis by host-inducible nitric oxide synthase. Cancer Res 63:3855-3859

Wyckoff J, Wang W, Lin EY, Wang Y, Pixley F, Stanley ER, Graf T, Pollard JW, Segall J, Condeelis J (2004) A paracrine loop between tumor cells and macrophages is required for tumor cell migration in mammary tumors. Cancer Res 64:7022-7029 